Trauma Berufskrankh 2007 - 9[Suppl 3]: S302-S307

DOI 10.1007/s10039-007-1307-3

Online publiziert: 1. November 2007

(c) Springer Medizin Verlag 2007

\section{T. Kälicke}

Chirurgische Klinik und Poliklinik, BG-Kliniken Bergmannsheil, Klinikum der Ruhr-Universtität Bochum, Bochum

\title{
Frakturen am Metatarsus
}

\section{Wann konservative, wann operative Behandlung?}

Die Frage „Wann ist eine konservative bzw. operative Frakturbehandlung sinnvoll?" ist im klinischen Alltag eines Traumatologen von zentraler Bedeutung. Im Folgenden möchte ich versuchen, Entscheidungshilfen bzw. -richtlinien für die Behandlung von Mittelfußfrakturen zu erstellen.

\section{Verletzungsmechanismen}

Allein der vorliegende Verletzungsmechanismus gibt oftmals schon entscheidende Hinweise, ob ein konservatives oder operatives Vorgehen indiziert ist. Als Verletzungsmechanismen, die eine Mittelfußfraktur verursachen können, sind die folgenden zu nennen:

- das direkte Trauma, das von der einfachen, undislozierten Fraktur bis hin zur ,crush injury“ führt,

- der Inversions-Avulsions-Mechanismus, dessen Folgen von der einfachen Avulsion der Basis von MFK V bis hin zur Lisfranc-Luxationsfraktur reichen,

- Überlastungen, die MFK-Frakturen zur Folge haben.

Wenn die Frage nach dem konservativen bzw. operativen Vorgehen beantwortet werden soll, müssen zunächst die entsprechenden Therapieziele definiert werden:

Ziel der Behandlung sollte eine schmerzfreie Belastungsfähigkeit des Fußes mit ungestörtem, physiologischem Gangbild sein. Dies sollte in einer sowohl für den Patienten als auch den Arzt akzeptablen - und das ist ein dehnbarer Begriff - Behandlungsdauer erreicht werden.

\section{Innen-Außen-Quer-Regel}

Um das Ziel der Belastungsfähigkeit zu erreichen, bedarf es auf der einen Seite der Integrität bestimmter anatomischer Strukturen und auf der anderen Seite einer Stabilität im Mittelfußbereich. Stichworte bezüglich der anatomischen Struk-

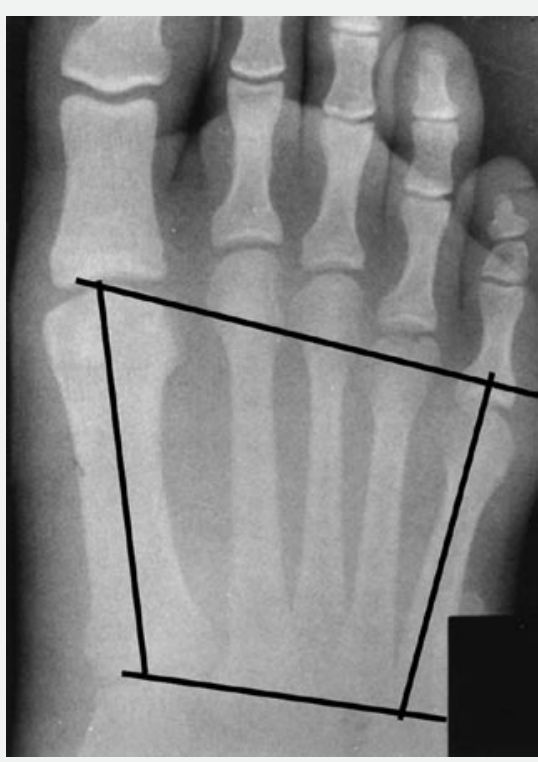

Abb. 1 \ Innen-Außen-Quer-Regel

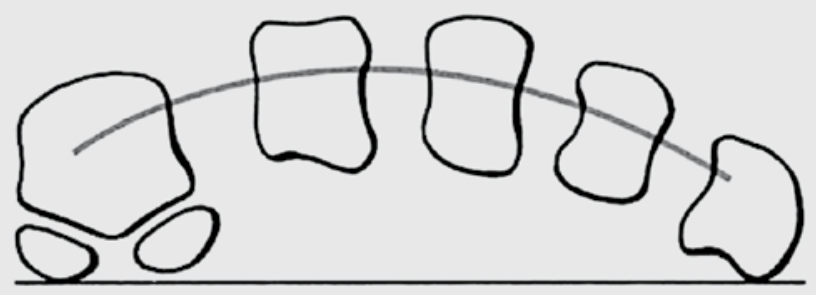

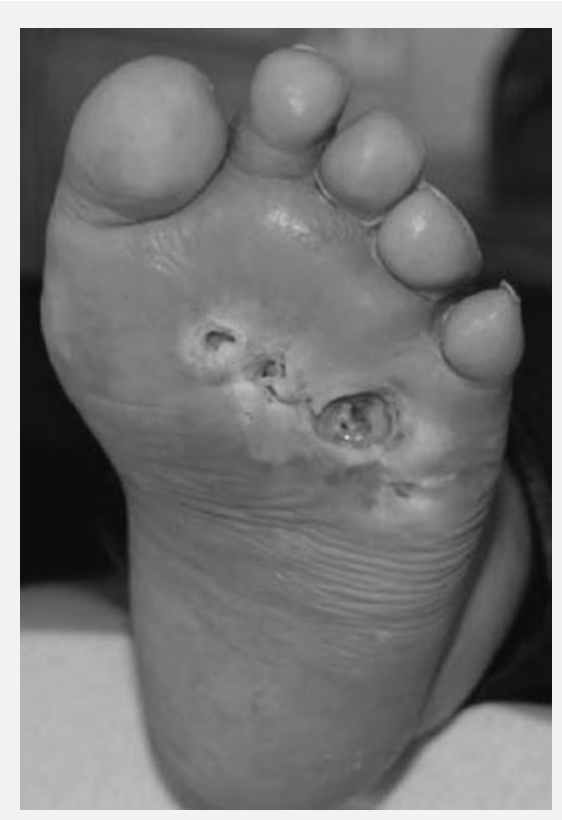

Abb. $3 \boldsymbol{\Delta}$ Druckulzera über den Köpfchen MFK II-V

turen sind die mediale und laterale Fußsäule mit dem Fußlängsgewölbe und das Fußquergewölbe („skyline“). Voraussetzung für eine adäquate Stabilität im Mittelfußbereich ist die Integrität der Lisfranc-Gelenklinie. Wenn ich mir bei jeder Fraktur am Mittelfuß diese Eckpfeiler vergegenwärtige, kann ich bereits fast

Abb. $2 \triangleleft$ Skyline 
immer festlegen: konservatives oder operatives Behandlungsregime.

Ich nenne das für mich die „Innen-Außen-Quer-Regel“ (• Abb. 1).

Dabei bezieht sich innen auf die mediale Säule, außen auf die laterale Säule. Die Integrität bzw. Rekonstruktion dieser Säulen ist Voraussetzung für einen physiologischen Gang mit schmerzfreier Belastungsfähigkeit. Quer gibt es zwei Linien, welche sorgfältig betrachtet werden müssen. Eine Linie verläuft quer distal: Hierbei handelt es sich um die sog. Skyline, d. h. das Fußquergewölbe (• Abb. 2). Eine frakturbedingte Veränderung des physiologischen Bogens kann unter anderem zu schmerzhaften Druckstellen und Ulzera führen (• Abb. 3). Die Untersuchung des Bogens sollte - wenn immer möglich - im Vergleich zur Gegenseite erfolgen, da die Ausprägung des Bogens individuell unterschiedlich ist und sein Ausmaß oftmals im Laufe des Lebens, insbesondere bei vorbestehendem Knick-Senk-Fuß, abnimmt. Die andere verläuft quer proximal: Hierbei handelt es sich um die LisfrancGelenklinie, die bei jeder Verletzung im Mittelfußbereich subtil untersucht werden muss, damit man nicht in eine falsche Behandlungsschiene gerät.

\section{Behandlungsdauer}

Ein weiterer Aspekt der Entscheidungsfindung „konservativ oder operativ“ ist die Behandlungsdauer. Diese sollte nicht unnötig in die Länge gezogen werden. Im Behandlungskonzept muss dabei unbedingt berücksichtigt werden, dass lange Ruhigstellungen zu Muskelatrophien, Muskeldysbalancen, Störungen der Propriozeption und Knochenentkalkung führen können, die dem eigentlichen Behandlungsziel einer schmerzfreien Belastungsfähigkeit des Fußes mit ungestörtem physiologischem Gangbild entgegenstehen.

\section{Anwendung der Innen-Außen-Quer-Regel}

Die folgenden Abbildungen zeigen verschiedene Anwendungsbeispiele der Innen-Außen-Quer-Regel:

MFK-I-Fraktur. Ein Beispiel dieser Fraktur ist in - Abb. 4a-d gezeigt:
- Innen: Die mediale Säule ist aufgrund einer Schaftfraktur von MFK I verkürzt und valgisiert

- Außen: Die laterale Säule ist intakt

- Quer distal: Skyline intakt

- Quer proximal: Lisfranc steht

Eine Operationsindikation ist für die mediale Säule gegeben, da nur deren anatomische Rekonstruktion eine spätere schmerzfreie physiologische Belastbarkeit des Fußes erwarten lässt. Die operative Versorgung erfolgte in diesem Fall durch zwei gekreuzte K-Drähte. Biomechanisch sinnvoller wäre sicherlich eine Plattenosteosynthese gewesen, aber wahrscheinlich haben dies die Weichteile nicht zugelassen. Denn MFK-I-Frakturen treten aufgrund des im Vergleich zu den MFK II$\mathrm{V}$ sehr starken kortikalen Knochens von MFK I meist nur bei hoher Gewalteinwirkung auf, meist einhergehend mit einem nicht unerheblichen Weichteilschaden.

MFK-I-Basisfraktur, intraartikulär mit Gelenkstufe sowie Lisfranc-Luxation. Eine Operationsindikation ist in dem in - Abb. 5 gezeigten Beispiel aufgrund der intraartikulären Gelenkstufe gegeben:

- Innen: Basisfraktur MFK I, intraartikulär mit Gelenkstufe

- Außen: Die laterale Säule ist intakt

- Quer distal: Skyline intakt

- Quer proximal: Anhand der Bilder nicht zu beurteilen

Da eine solche Fraktur eigentlich nicht durch ein direktes Trauma hervorgerufen werden kann, sondern vielmehr ein Inversions-Avulsions-Mechanismus für die Verletzung postuliert werden muss, ist bei der klinischen Untersuchung bzw. intraoperativ ein besonderes Augenmerk auf die Lisfranc-Gelenklinie zu richten. Intraoperativ zeigte sich hier neben der Basisfraktur MFK I, welche mit 2 Schrauben versorgt wurde, eine Instabilität in der Lisfranc-Reihe, mit entsprechender temporärer Transfixation des MFK I gegen das Os cuneiforme mediale.

„Rahmensprengung“. Die Sprengung eines gesamten Rahmens ist aus 0 Abb. 6 ersichtlich:

- Innen: Luxationsfraktur MFK I
Trauma Berufskrankh 2007 - 9[Suppl 3]: S302-S307

DOI 10.1007/s10039-007-1307-3

C) Springer Medizin Verlag 2007

T. Kälicke

\section{Frakturen am Metatarsus. Wann konservative, wann operative Behandlung?}

\section{Zusammenfassung}

Die Frage „Wann ist eine konservative bzw. operative Frakturbehandlung sinnvoll?" ist im klinischen Alltag eines Traumatologen von zentraler Bedeutung. Im vorliegenden Beitrag wurden Entscheidungshilfen bzw. -richtlinien für die Behandlung verschiedener Mittelfußfrakturen erstellt. Insbesondere die „Innen-Außen-Quer-Regel", bei der die genaue Beobachtung von 4 Linien - der medialen Säule, der lateralen Säule, der sog. Skyline (Fußgewölbe) und der Lisfranc-Gelenklinie im Vordergrund steht, hat sich als Entscheidungshilfe bewährt.

\section{Schlüsselwörter}

Mittelfußfraktur · Innen-Außen-Quer-Regel . Verletzungsmechanismen .

Operationsindikation

\section{Metatarsal fractures. Nonoperative vs operative treatment: when to do which?}

\section{Abstract}

The question "When is conservative and when is operative fracture treatment reasonable?" is of central importance in the day-today clinical practice of an orthopaedic surgeon. The present paper is intended to give some guidance and directives for decision making in the treatment of metatarsal fractures. The medial-lateral-transverse rule in particular, according to which precise observation of four lines - the medial column, the lateral column, the so-called skyline (plantar arch) and the Lisfranc joint line - is paramount, has proved its worth as an aid to decision making.

\section{Keywords}

Metatarsal fracture $\cdot$ Medial-lateral-transverse-rule $\cdot$ Mechanisms of injury $\cdot$ Indications for surgery 


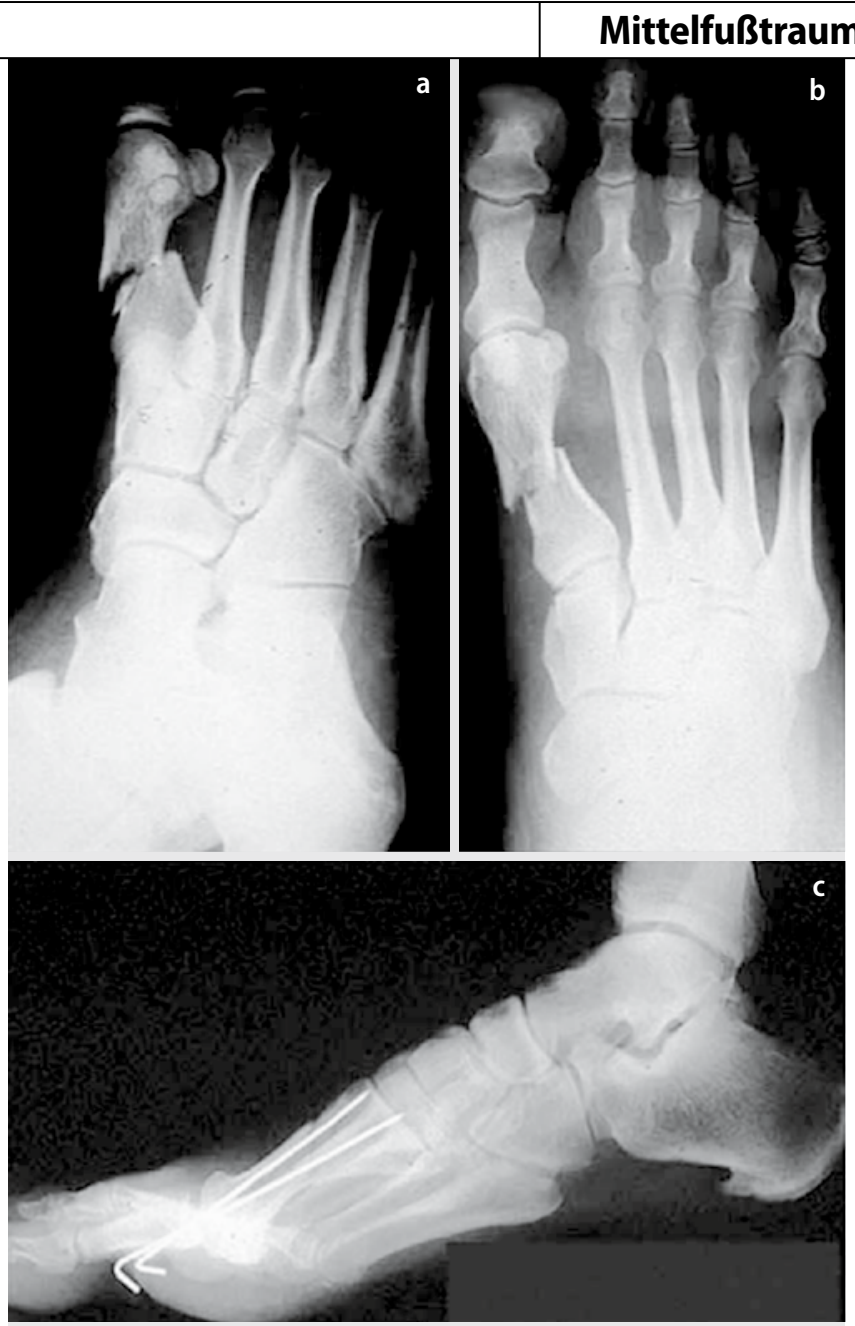

b

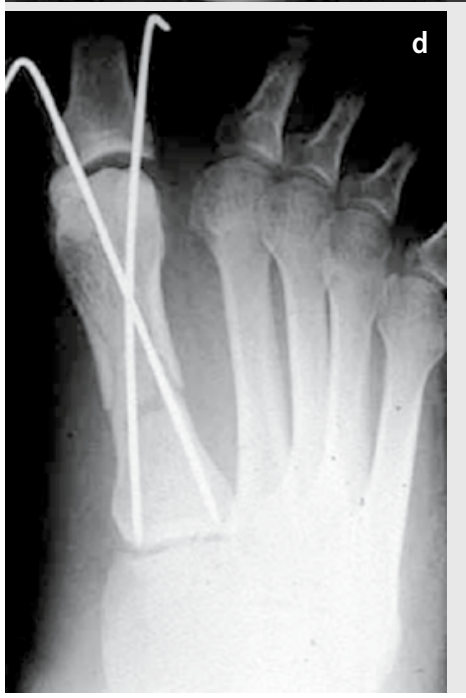

Abb. $4<$ a, b MFKI-Fraktur; c, d Versorgung mit K-Drähten

- Außen: Um mehr als Schaftbreite dislozierte MFK-V-Schaftfraktur mit Verkürzung und begleitenden Schaftfrakturen von MFK II-IV

- Quer distal: Verbreiterung

- Quer proximal: Luxation

Hier müssen die mediale und die laterale Säule rekonstruiert und die Lisfranc-Rei-
MFK-I-Fraktur, verkürzt und valgisiert, mit Halsfrakturen MFK II-IV. Die

- Abb. 7a und b fokussieren auf die häufigen Frakturen der MFK II-IV.

- Innen: MFK-I-Fraktur mit Verkürzung und Valgisierung. Eine Operationsindikation ist klar gegeben

- Außen: Die laterale Säule ist intakt

- Quer proximal: Anhand des Bildes nicht sicher zu beurteilen

- Quer distal: Halsfrakturen MFK IIIV

Laut Literatur ist bei diesen Frakturen eine Operationsindikation ab einem Achsknick $>10^{\circ}$ bzw. einer Dislokation $>3^{-}$ $4 \mathrm{~mm}$ gegeben [1]. Dies ist aber oftmals röntgenologisch nur schwer zu beurteilen. Die Entscheidung, ob operativ oder konservativ vorgegangen wird, richtet sich im Wesentlichen nach der SkylineAufnahme. Sinken die MF-Köpfe im Vergleich zur Gegenseite ab, wird dem Patienten die Operation empfohlen. Der alternative Versuch, geschlossen zu reponieren und das Repositionsergebnis im Gips retiniert zu halten, gelingt aufgrund des Zugs der intrinsischen und extrinsischen Fußmuskulatur nur selten.

Unzureichende Röntgendiagnostik bei unverschobenen Frakturen. Die

- Abb. 8 zeigt unverschobene Frakturen der MFK II-IV. Diese können natürlich wie fast alle unverschobenen Mittelfußfrakturen konservativ behandelt werden. Die Abbildung zeigt jedoch das Beispiel einer Röntgenaufnahme, die nicht akzeptiert werden kann, da eine Beurteilung Innen-Außen-Quer hier nicht möglich ist. In diesem Fall könnte es sich z. B. auch um einen Inversions-Avulsions-Unfallmechanismus handeln, bei dem noch eine Avulsion des MFK V denkbar ist, die operativ angegangen werden müsste. Es muss also immer auf eine qualitativ hochwertige Röntgendiagnostik geachtet werden.

Halsfraktur MFK V mit Verkürzung und Versatz um halbe Schaftbreite in Kombination mit Frakturen der MFK II-IV. In

- Abb. 9 ist die Kombination verschiedener Frakturen zu erkennen:

- Innen: Die mediale Säule ist intakt 

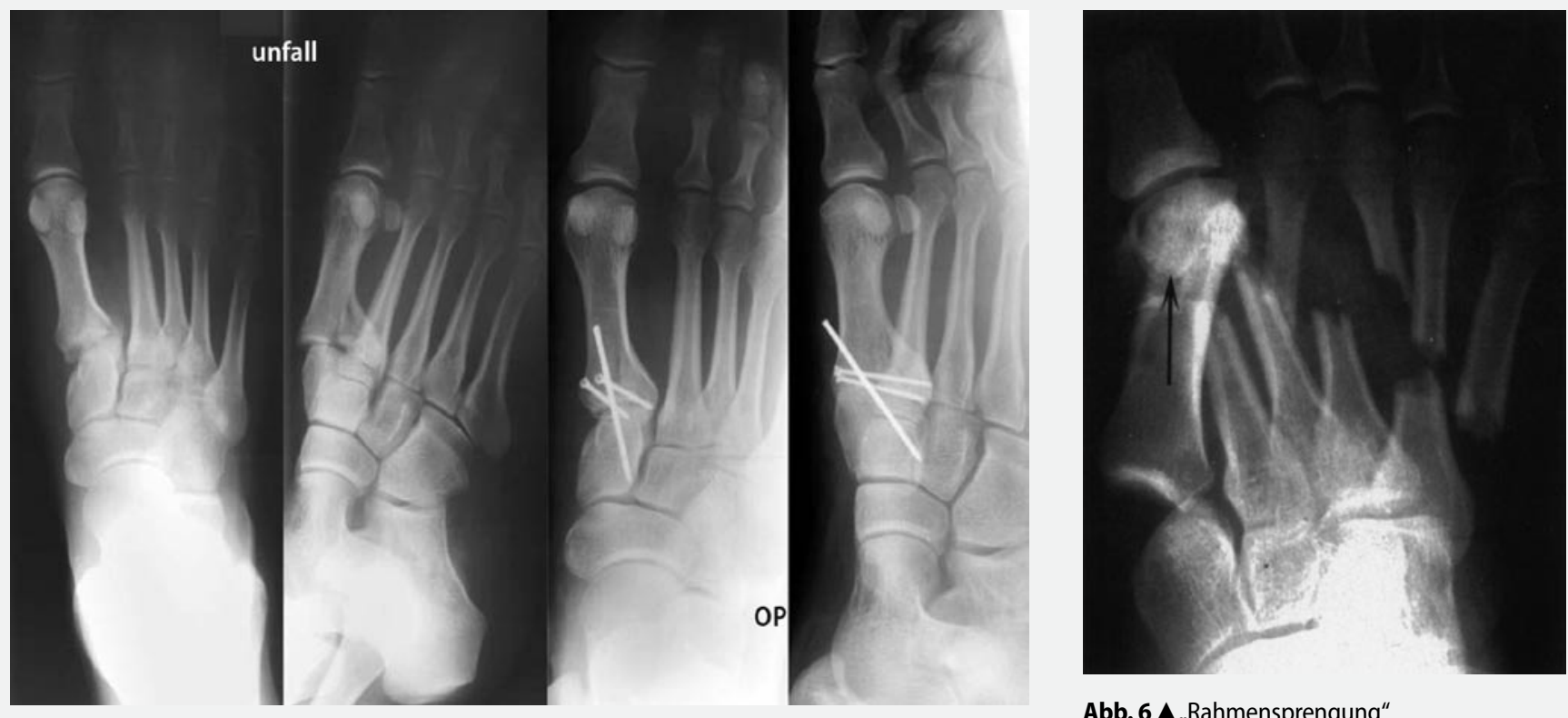

Abb. $6 \Delta_{\text {", Rahmensprengung“ }}$

Abb. 5 \ MFK-I-Basisfraktur, intraartikulär mit Gelenkstufe sowie Lisfranc-Luxation
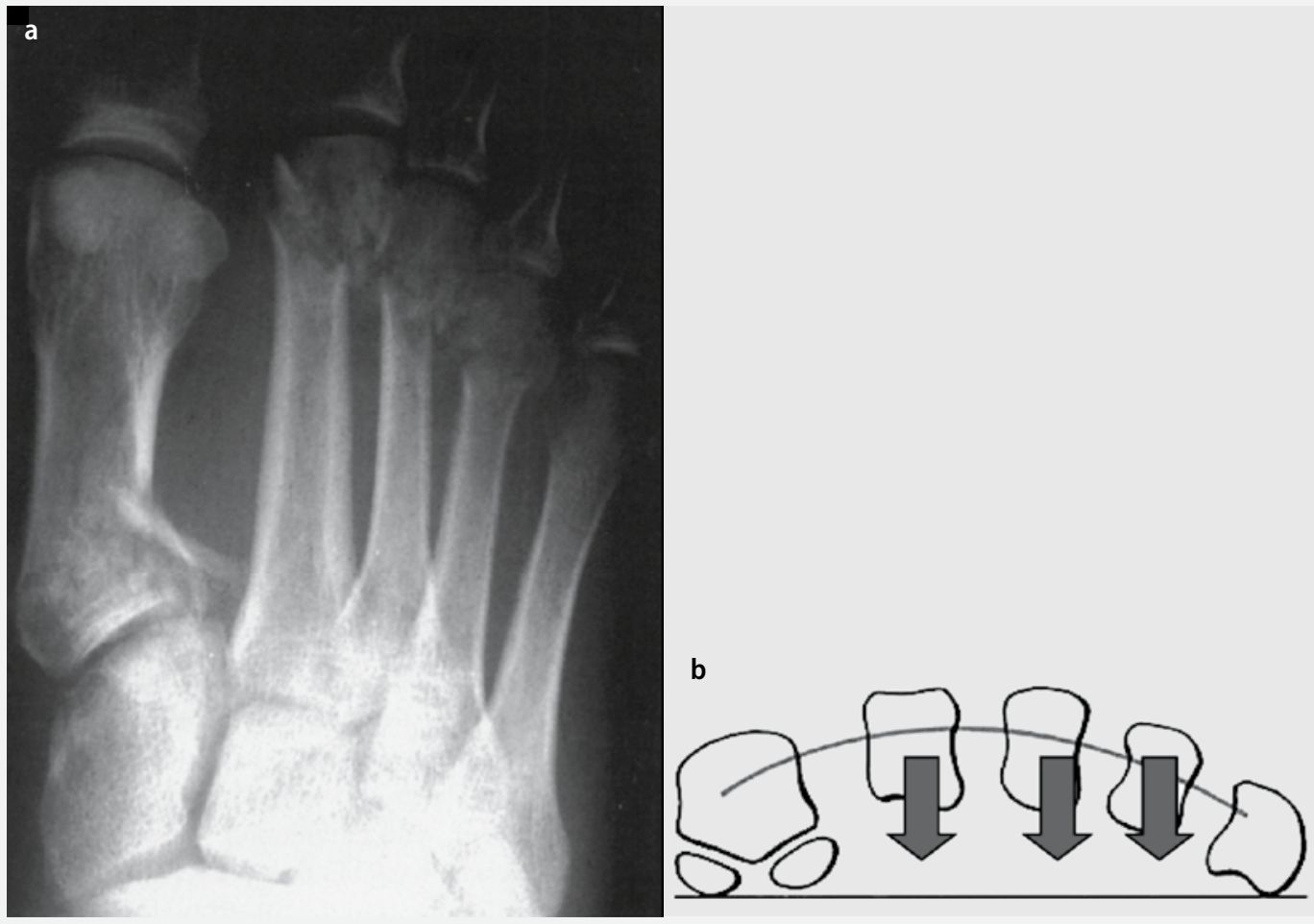

\begin{abstract}
Abb. $7<$ a MFK-I-Fraktur, verkürzt und valgisiert, mit Halsfrakturen der MFK IIIV; b Gefahr des plantaren Abkippens der MF-Köpfchen II-IV
\end{abstract}

- Außen: Halsfraktur MFK V mit Verkürzung und Versatz um halbe Schaftbreite

In Kombination mit den Frakturen MFK II-IV droht ein starker Valgus des Fußes, daher Operationsindikation mit Rekonstruktion der lateralen Säule gegeben. Hierdurch werden sich II-IV wahrscheinlich gut einstellen und müssen nicht operativ angegangen werden, wobei aber die Skyline im Auge behalten werden muss.

Die Halsfraktur eines MFK disloziert klassischerweise nach lateral/plantar. Nach erfolgter Reposition lässt sich das Repositionsergebnis nur schlecht retiniert halten, sodass operativ vorgegangen werden sollte, wenn es im Vergleich zur Gegenseite durch die Dislokation zu einer relevanten Kompromittierung der Skyline kommt.
Bei der intraartikulären Kopffraktur eines MFK ist die Operation laut Literatur bei einer Gelenkstufe >1-2 mm gegeben [1].

Bei den Basisfrakturen MFK II-IV liegt eine gänzlich andere Situation als bei der Basisfraktur MFK I vor. Aufgrund der starken Bandstrukturen handelt es sich meist um undislozierte Frakturen, die konservativ behandelt werden können bzw. sollen. Allerdings muss vorher 


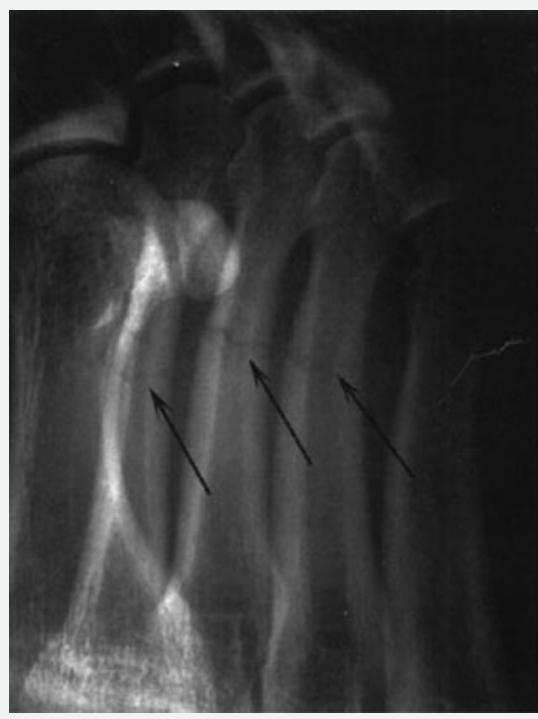

Abb. $8 \Delta$ Insuffiziente Röntgendiagnostik bei unverschobenen MFK-Frakturen

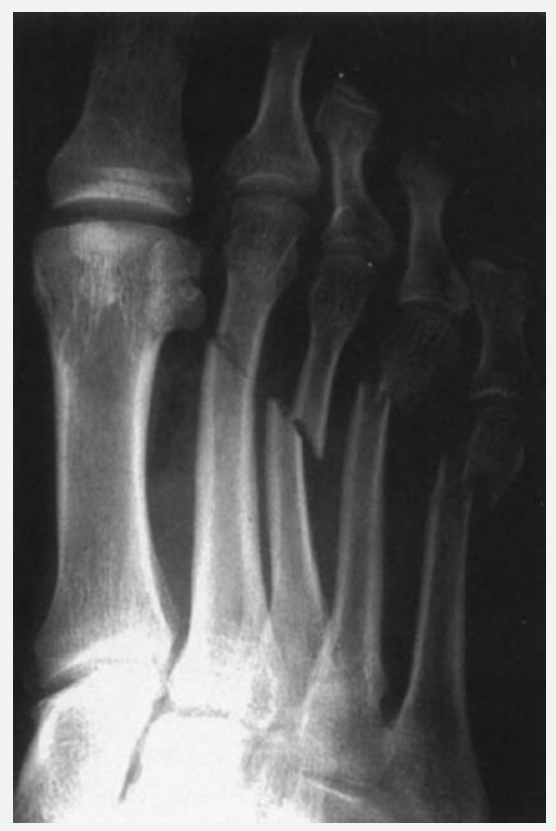

eine Instabilität, $d$. h. eine Lisfranc-Verletzung ausgeschlossen werden.

\section{MFK-V-Frakturen}

Im Folgenden werden anhand einiger Beispiele die häufigen, isolierten MFK-VFrakturen diskutiert:

Spiralbruch MFK V. In $\bullet$ Abb. 10 ist der klassische Spiralbruch MFK V im Rahmen eines Supinationstraumas abgebildet. Ein solcher Bruch muss nicht operiert werden! Diese Fraktur heilt aufgrund der großen knöchernen Kontaktfläche eigentlich immer. Es sollte aber eine Skylineauf-
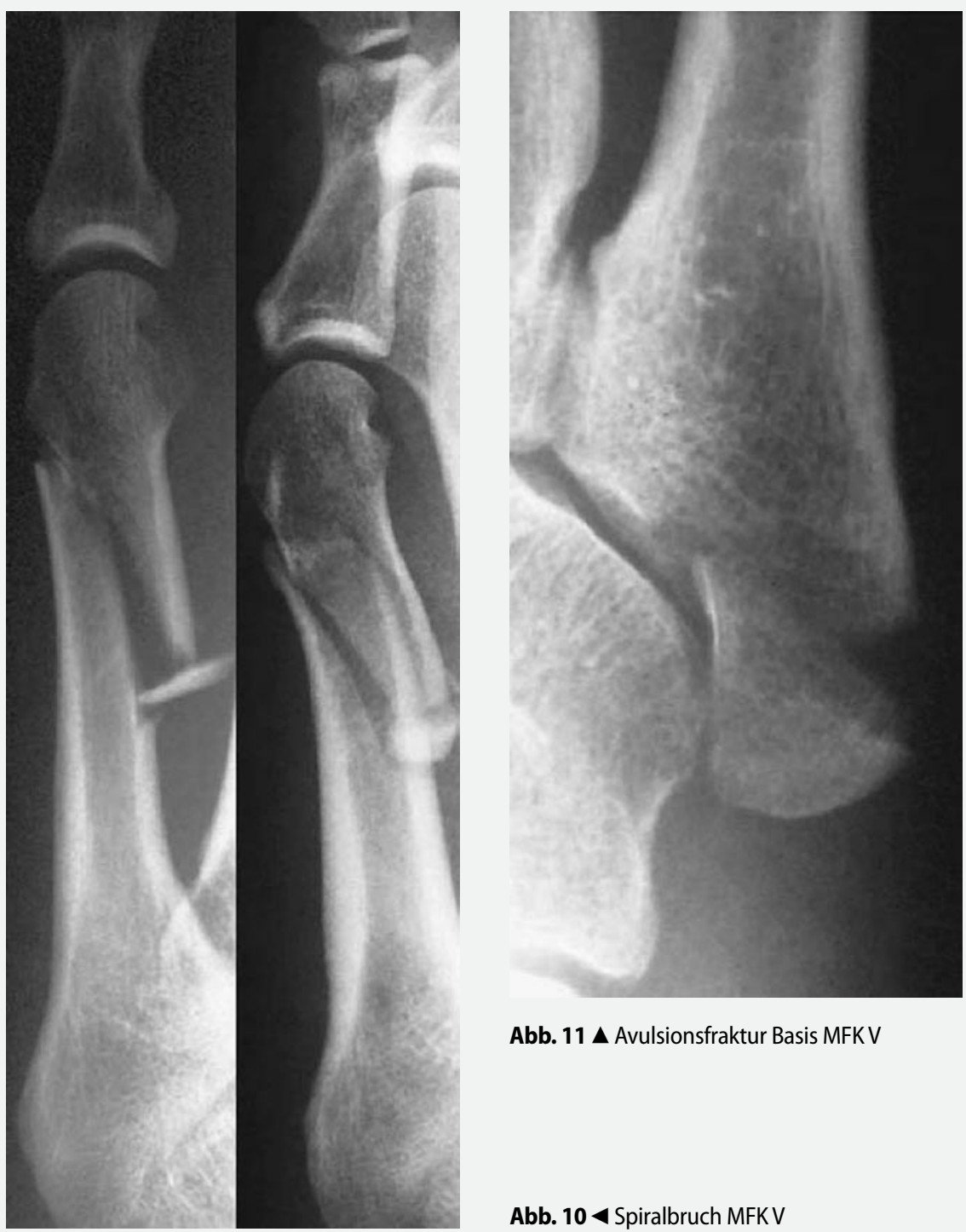

Abb. $11 \Delta$ Avulsionsfraktur Basis MFKV

Abb. $9 \varangle$ Halsfraktur des MFK V mit Verkürzung und Versatz um eine halbe Schaftbreite; in Kombination mit den Frakturen der MFK II-IV droht ein starker Valgus des Fußes

nahme gemacht werden, um einen eventuellen Drehfehler, der später Schmerzen verursachen kann, auszuschließen.

MFK-V-Basisfraktur. Die • Abb. 11 zeigt eine typische MFK-V-Basisfraktur (auch Avulsionsfraktur genannt). Alle aktuellen Arbeiten geben als Indikation für eine Operation eine Dislokation um $>2 \mathrm{~mm}$ und/oder Gelenkflächenbeteiligung >30\% an. Es handelt sich hierbei allerdings jeweils nur um Evidenzlevel-V-Arbeiten, d. h. Expertenmeinungen. „Harte Fakten“ liegen dazu nicht vor.

\section{Jones-Fraktur}

Bei der sog. Jones-Fraktur handelt es sich um eine transversale Fraktur am metadiaphysären Übergang von MFK V, die typischerweise etwa $1,5 \mathrm{~cm}$ distal der Basis des MFK V gelegen ist. Sie ist fast immer undisloziert. Die Therapie dieser undislozierten Jones-Frakturen wird in der Literatur immer noch kontrovers diskutiert. Coughlin et al. [1] propagieren ein konservatives Vorgehen mit 6- bis 8-wöchiger Gipsschuhruhigstellung unter Abrollbelastung. Sie verweisen hierbei auf ein großes Zeitfenster zwischen klinischer Heilung und radiologisch nachgewiesener knö- 
cherner Durchbauung. Letztere folgt der klinischen Heilung oftmals erst Wochen oder sogar Monate später. Quill [3] kommt in einer Metaanalyse der relevanten Literatur zu dem Ergebnis, dass nach konservativem Vorgehen Pseudarthrose- bzw. Refrakturraten von etwa $30 \% \mathrm{zu}$ verzeichnen sind, wenn man nur lange genug nachuntersucht. Er zieht daraus den Schluss, dass die primär operative Versorgung einer undislozierten Jones-Fraktur zumindest keinen Fehler darstellt. Einigkeit besteht in der Literatur darüber, dass die dislozierte Jones-Fraktur offen eingerichtet und intern fixiert werden muss.

\section{Stressfraktur}

Abschließend noch eine kurze Bemerkung zu den Stressfrakturen der Mittelfußknochen, die meist bei Leistungssportlern oder Rekruten auftreten. Gestatten Sie mir als ehemaligem Leistungssportler, der sich 3-mal eine Stressfraktur zuzog, hier folgende Evidenzlevel-V-Aussagen:

1. Eine Operation ist nicht erforderlich.

2. Eine spezielle Ruhigstellung ist nicht erforderlich.

3. Es ist alles erlaubt, was nicht schmerzt (d. h., die vormalige Belastungsintensität im Training muss zwingend reduziert werden, was dem Athleten allerdings oftmals nur schwer vermittelbar ist).

Vom Athleten wird in diesem Zusammenhang die alles entscheidende Frage an den Arzt gerichtet werden: „Kann ich durch eine Operation schneller wieder das Training aufnehmen und an Wettkämpfen teilnehmen?" Gehrmann [2] beantwortet diese Frage im aktuellen „Current Concepts Review“ der Zeitschrift Foot and Ankle International ausweichend damit, dass eine Operation theoretisch eine schnellere Rückkehr eines Athleten auf sein vorheriges Trainingsniveau ermöglichen sollte. „Harte Fakten“ existieren aber wiederum nicht.

\section{Fazit}

Zusammenfassend können folgende Richtlinien aufgestellt werden:

- Bei Frakturen am Metatarsus sollte im Rahmen der Entscheidungsfindung
„Wann konservatives, wann operatives Vorgehen?" die Innen-AußenQuer-Regel angewendet werden.

- Frakturen des MFK I müssen (fast ausnahmslos) operiert werden.

- Besondere Aufmerksamkeit sollte bei Frakturen der MFK II-IV der Aufnahme der Skyline geschenkt werden.

- Bei Avulsionsfrakturen des MFK V ist bei einer Dislokation $>2 \mathrm{~mm}$ und/ oder Gelenkflächenbeteiligung $>30 \%$ eine Operation indiziert.

- Bei einer Jones-Fraktur des MFK V ist ein konservatives Vorgehen zu empfehlen (nur die seltene dislozierte Jones Fraktur wird operiert).

- An eine Mitbeteiligung der LisfrancGelenklinie sollte grundsätzlich gedacht werden.

- Bei Stressfrakturen der MFK ist konservatives Vorgehen zu empfehlen. Es ist alles erlaubt, was nicht schmerzt.

\section{Korrespondenzadresse \\ PD Dr. T. Kälicke \\ Chirurgische Klinik und Poliklinik, BG-Kliniken Bergmannsheil, Klinikum der Ruhr-Universtität Bochum, Bürkle-de-la-Camp-Platz 1, 44789 Bochum tkaelicke@compuserve.de}

Interessenkonflikt. Keine Angaben

\section{Literatur}

1. Coughlin MJ, Mann RA, Saltzman CL (2006) Surgery of the foot and ankle. $8^{\text {th }}$ edition, Mosby, St. Louis

2. Gehrmann RM, Renard RL (2006) Current concepts review: stress fractures of the foot. Foot Ankle Int 27: 750-757

3. Quill GE (1995) Fracture of the proximal fifth metatarsal. Orthop Clin North Am 26: 353-361 